\title{
Simulation Models for Management Solutions when Implementing Innovative Technologies
}

\author{
Irina Klavsuts $^{1 *}$, \\ ${ }^{1}$ Department of Management, Novosibirsk State Technical University, Russia
}

\begin{abstract}
The object of empirical research is a company that develops, produces and implements innovative patented technology for managing electricity consumption, naturally fitting into the concept of Smart Grid. The author has developed simulation models for predicting the behavior of an innovative technology market. A paradigm of simulation modeling - system dynamics - is investigated and implemented. In this work, hypotheses about the origin and significant development of communications between market segments are put forward and investigated. The introduction of such links in the developed simulation models is completely natural and technically simple, but their use requires additional research. Mass simulation runs of the developed models with the introduction of new market relations and parameters make it possible to more accurately understand the market behavior mechanisms of the innovative patented technology, use these mechanisms to develop models, build sound long-term forecasts, develop realistic production plans, improve management efficiency through the introduction of the innovative technology.
\end{abstract}

\section{Introduction}

Currently, the concept of smart electric networks -Smart Grid is actively developing, which is the basis and key component of strategies and projects for improving energy efficiency. Smart Grid is a set of technical and economic measures that include monitoring electric consumption, dynamic management of electric networks, regulation of electricity demand in power supply systems - Demand Response, and management of electricity consumption Demand Side Management (DSM). To form and implement strategies for the development and implementation of innovative technologies in Smart Grid systems, it is necessary to develop and evaluate the effectiveness of management decisions made.

The research is aimed at developing and improving the methodology of strategic planning based on simulation modeling for making management decisions on the production and implementation of a fundamentally new technology for managing energy consumption - a patented method and devices for managing energy consumption that increase the energy efficiency of Smart Grid systems. The method and device are patented in the Russian Federation, the USA, CIS, EC, ASEAN, Ukraine and other countries on the basis of the international application for the invention "Alternating voltage stabilizer with protection

\footnotetext{
* Corresponding author: ira.klavsuts@gmail.com
} 
elements (embodiments)", published in accordance with the patent cooperation act (PCT) by the world intellectual property organization, which indicates the emergence of a fundamentally new technology [1-7].

For wide implementation of the innovative technology in 2009, in Novosibirsk, Russian Federation, the research and production enterprise "AVEC" LLC was organized, which is currently the only one in the world that produces and improves innovative patented devices for controlling electric energy consumption, called "AC voltage normalizers", under the trademark NORMEL ${ }^{\mathrm{TM} \otimes}[8]$.

NORMEL $^{\mathrm{TM} \mathbb{C}}$ AC voltage Normalizer devices are connected to the electrical inputs of buildings, structures, industrial equipment, street lighting, i.e. any electric energy consumers in any country of the world.

The application of the method and device allows saving financial and other resources by reducing consumption of electric energy (by the meter) from $7 \%$ to $24 \%$ depending on the nature of electrical load, based on voltage control of power consumers. In addition, by improving the quality of electric energy in accordance with the international standard on quality of electric energy, for example, GOST 32144-2013 and the identical European standard EN 50160: 2010 [9, 10]. It results in significantly increased service life, and the absence of interruptions in the operation of electrical equipment, and therefore, the absence of faulty production. RPE AVEC LLC together with NSTU conducts research and R \& D, which is aimed at the widespread introduction of innovative methods and devices NORMEL $^{\mathrm{TM} \circledast \mathcal{O}}$ in the electric power systems of the Russian Federation, the CIS, the EU and other countries of the world. Besides, the methods and devices are used at the departments of Theoretical and Applied Informatics, Automated electric power systems, in the center for testing devices for monitoring and controlling modes of electric power systems and in the Scientific and educational center of "Intelligent information technologies in business".

Research and years of experience of using the method and devices by numerous enterprises, institutions, households, buildings, structures, street lighting in Russia and CIS show that the effect of the use of technology by direct consumers. This effect is also manifested in the generating, electric network and power marketing companies, i.e. in the electric energy system overall, including the source of electricity -power stations.

Research and experience in implementing innovative technology in various countries show that the systematic mass application of this technology in the world's electrical systems within the framework of the Smart Grid concept allows to effectively manage electric energy consumption by improving its quality, which significantly increases their energy efficiency.

The total economic effect of the system application of the innovative NORMEL ${ }^{\text {TMBC }}$ technology in the world's electric power systems by reducing electricity consumption and improving its quality can reach more than 50\% savings in financial and other resources related to the production, transmission and consumption of electric energy, as well as all related environmental effects. There are no similar devices in the world, which have such an effect [11-18].

When implementing innovative technology, research and production enterprises inevitably face practical issues and challenges in developing and making effective management decisions that improve the accuracy and quality of investment planning, production management, implementation and sales based on long-term forecasting of the market behavior of potential users of these technologies.

The author has developed a simulation model for predicting the behavior of the market of innovative technology, based on the concept of Frank M. Bass [19] about the division of market agents into innovators and imitators functioning under the influence of different factors. The awareness of these factors and regulated parameters allows identifying and effectively managing a long-term process of implementation. 
We used the paradigm of simulation modeling - system dynamics, proposed by G. Forrester [20]. The solution to the problem of implementing innovative products using system dynamics is based on Frank's work. The simplest working model of this problem is implemented and available for research in the Any Logic simulation system [21, 22].

The system-dynamic model is studied on the example of the introduction of innovative patented technology for managing electricity consumption in isolated segments of the Novosibirsk market - medium and small enterprises. The model is described in detail and the results are interpreted.

In conclusion, we present the results of the work, formulate management decisions on the introduction of innovative technology to groups of market segments, the features and limitations of the Bass model, its implementation in AnyLogic, and recommendations for their use.

\section{Model of system dynamics of innovative technology implementation in an isolated market}

Let's consider a methodological approach to the development of the Bass model on the example of a system-dynamic model of introducing innovative technology for managing energy consumption to the market of potential consumers of this technology in Novosibirsk city in conditions when the studied innovative enterprise enters a new market, introduces new business models, strategies, and communication processes. Traditional examples of implementing the model of F. Bass distribution of innovations describe the behavior of only one market, and, moreover, it's a retail market [23].

In contrast to the traditional model, in the case under consideration, the isolated market may include retail consumers as well as wholesale consumers, and on different levels, at that. Among wholesale consumers, the segments of medium and small enterprises occupy an important place. Other segments in the near future may include consumers of electric energy from large businesses, various industries, etc.

In this paper, the research is still limited to the segments of medium and small enterprises. At the moment, up to 5,000 small and 300 medium-sized enterprises can act as potential clients of the innovative technology in Novosibirsk [the assessment is based on the source: Small and medium-sized enterprises of Novosibirsk [24].

Let's start with a discussion of the system dynamics model.

The concept of system dynamics is "centralized". It is distracted from the study of the behavior of each individual element of the system, in our case, each specific enterprise. At the same time, general patterns of system behavior are assumed to be known. The structure of various types of relationships and model elements - accumulators, flows, factors, parameters, and variables - is important. The relationships determine the dynamics of the behavior of the entire system, which allows one to predict its development. And even for innovative technologies, when there is still no data or there is insufficient retrospective data.

Let's first consider the application for each individual sector (medium and small enterprises) of the simplest model of system dynamics, focused on typical markets, presented by the developers of AnyLogic. The traditional model of system dynamics is shown in Figure 1 . 


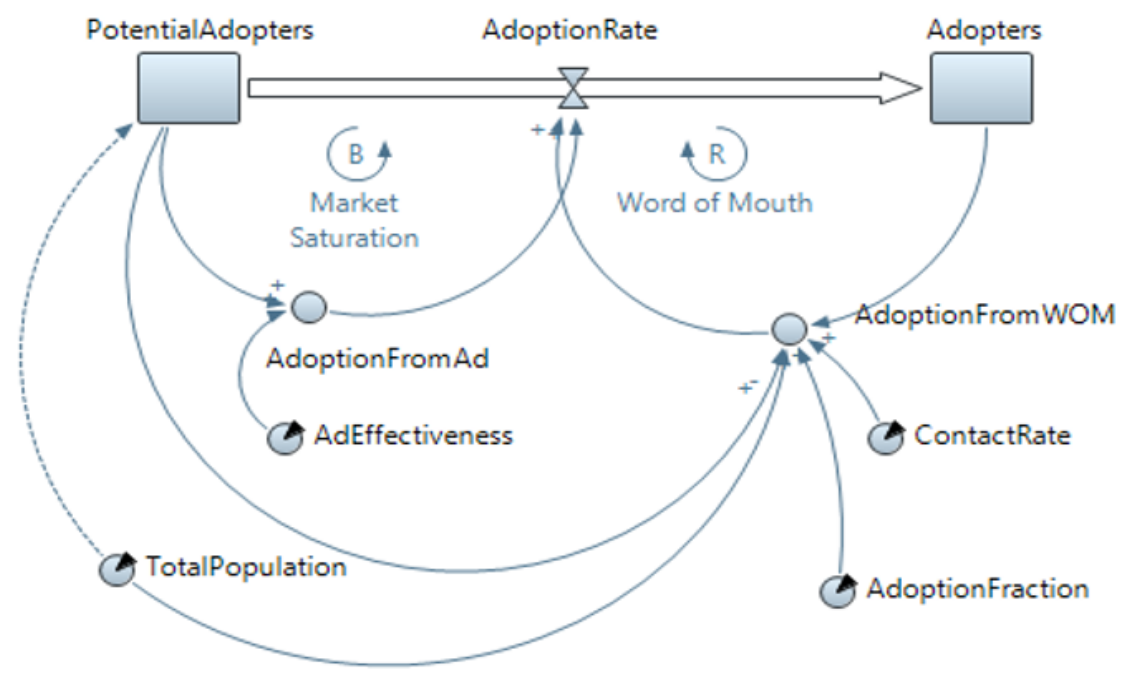

Fig. 1. Traditional system dynamics model.

Model designations in Fig. 1:

PotentialAdopters - storage device. The total number of potential users at the current point in the model time. The initial value is set by the TotalPopulation parameter.

Adopters- storage device. The number of users who have set the Normalizer at the current time in the model time. At the beginning of the experiment, this number is zero: the storage device is empty.

TotalPopulation - parameter. The total number of potential users of the innovative technology.

AdoptionRate - the flow (speed) of innovative technology implementation.

MarketSaturation - MarketSaturation-negative balancing (self-correcting) feedback. The result of direct influence of marketing and advertising products on potential users of innovative technology.

AdoptionFromAd - dynamic variable. Factor. Advertising.

AdEffectiveness - parameter. Effectiveness of advertising.

WordofMouth - amplifying feedback. Implementation under the influence of "word-ofmouth".

AdoptionFromWOM - dynamic variable. Factor. "Word of mouth".

ContactRate - parameter. Frequency of contacts per year of potential users.

AdoptionFraction - parameter. The power of persuasion of a client who has implemented an innovative technology, the influence on the "word of mouth" factor.

In the presented model, the connection between storage devices is a stream of implementation of innovative technology (normalizing devices) AdoptionRate, which converts Potential Adopters into users of innovative technology - Adopters. The factors that affect storage and the flow in the presented model are the system of directed information influence AdoptionFromAd and intra-market communications - Adoption FromWOM.

The regulated parameter for the AdoptionFromAd factor is its efficiency-Adeffectivity (a typical level of 0.011 was adopted on the recommendation of the developers), and for the intra - market communication factor AdoptionFromWOM - the population size TotalPopulation (300 medium and 5 thousand small enterprises), the frequency of contacts ContactRate, (in this example, 100 contacts per year among the participants of Word of Mouth, when the information about the innovative technology is received from the lips of 
both potential users and users of innovative technology) and the persuasive power of AdoptionFraction (0.015).

The first MarketSaturation feedback in the model is negative, balancing, and self-correcting. Enhancing the AdoptionFromAd factor increases the number of Adopters and reduces the number of potential users (PotentialAdopters). This limits the growth of the factor and leads to its decrease. The second feedback in the model is also balancing: the increase in Adopters under the influence of intra-cluster communications AdoptionFromWOM increases the number of Adopters and reduces the number of PotentialAdopters. This also limits the growth of the AdoptionFromWOM factor and leads to its decrease. The third feedback in the model is positive, reinforcing. Increasing implementations of normalizers under the influence of AdoptionFromWOM increases the number of Adopters. This leads to an even greater increase in AdoptionRate under the influence of AdoptionFromWOM, and to a decrease over time in the share of influence of the system of directed information influence on the stream AdoptionRate.

The stream of implementations of the normalizers AdoptionRate is set as a time derivative of PotentialAdopters with a minus sign and Adopters with a plus sign, as in the expression (1)

$$
\begin{gathered}
\frac{d(\text { PotentialAdopters })}{d t}=- \text { AdoptionRate } \\
\frac{d(\text { Adopters })}{d t}=\text { AdoptionRate }
\end{gathered}
$$

The model assumes the following assumptions:

1. The system of directed information influence and intra-market communications affect the AdoptionRate additively and with the same weight, as shown in the expression (2)

$$
\text { AdoptionRate }=\text { AdoptionFromAd }+ \text { AdoptionFromWOM }
$$

2. The flow of implementations of normalizers under the influence of the system of directed information influence is the product of the parameters of the effectiveness of the system of directed information influence and the number of potential consumers, as is presented in the expression (3)

$$
\text { AdoptionFromAd }=\text { AdEffectiveness } \times \text { PotentialAdopters }
$$

3. The flow of implementations of Normalizer devices under the influence of intra-cluster communications AdoptionFromWOM is the product of the number of implementing Adopters, the number of contacts ContactRate, the persuasive power AdoptionFraction, and the share of PotentialAdopters in the TotalPopulation, as is presented in the expression (4)

$$
\begin{aligned}
\text { AdoptionFromWOM } & =\text { Adopters } \times \text { ContactRate } \times \text { AdoptionFraction } \times \\
& \times \text { PotentialAdopters } / \text { TotalPopulation }
\end{aligned}
$$

All the above formulas (1) - (4) are used in AnyLogic when forming the system dynamics model shown in Figure 1.

The initial data for modeling is summarized in Table 1 . 
Table 1. Data for modeling the implementation of innovative technology in the market of Novosibirsk city.

\begin{tabular}{|c|c|c|c|c|}
\hline Market sector & TotalPopulation & AdEffectiveness & ContactRate & AdoptionFraction \\
\hline $\begin{array}{c}\text { Middle-sized } \\
\text { enterprises }\end{array}$ & 300 & 0.011 & 100 per year & 0.015 \\
\hline Small enterprises & 5000 & 0.011 & 100 per year & 0.015 \\
\hline
\end{tabular}

The current instantaneous results of the experiment are continuously displayed on all elements of the system dynamics model, which is inconvenient for presentation in printed form in static Figure 1 . Therefore, the model has the ability to generate dynamically changing schedules for the implementation of innovative technology.

On the left graph in Fig.2 the dynamics of implementation in the segment of medium-sized enterprises in Novosibirsk of the Russian Federation at the end of the experiment is presented. On the right graph in Fig. 2 the dynamics of modeling results is presented in the segment of small enterprises.
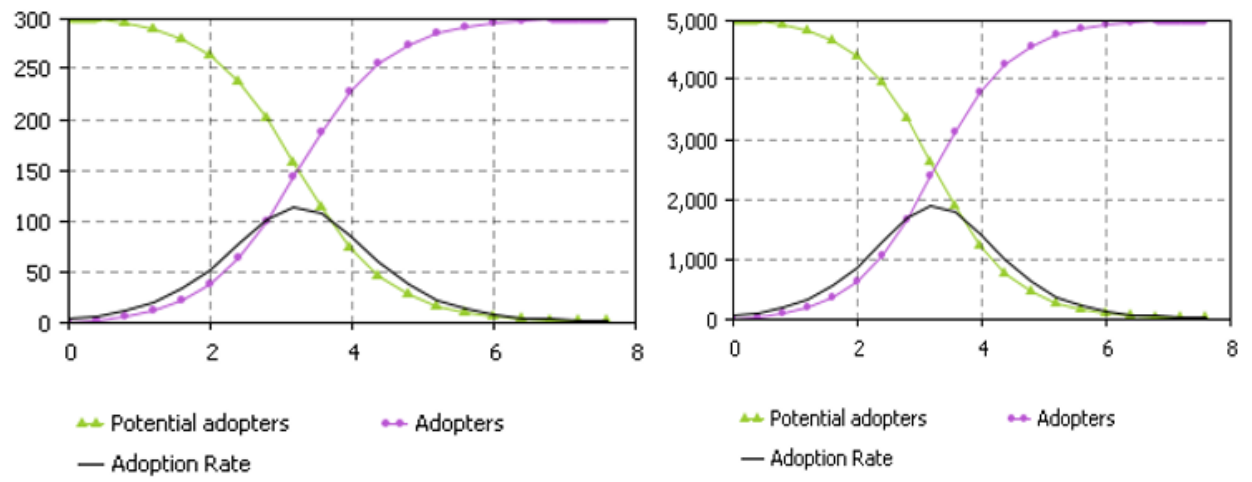

Fig. 2. Results of system-dynamic modeling of medium-sized (left graph) and small (right graph) enterprises

In Fig. 2 it can be seen that the number of Adopters (that have implemented innovative technology) in both segments is growing from zero at the start of the project to a maximum (Total Population) by the end of the eighth year. The growth curve has a symmetrical S-shape relative to the inflection point. The number of PotentialAdopters, on the contrary, falls from the maximum (TotalPopulation) to zero by the end of the eighth year. The implementation flow AdoptionRate has a bell-shaped appearance and reaches its maximum at the time corresponding to the inflection point of the S-shaped curves of PotentialAdopters and Adopters. For a manufacturer, this means that if, for example, they plan to mainly provide the innovative device to the market sector in about 8 years, as in the example shown, then with the selected model parameters, their production program should correspond to the curves shown in Fig. 2. Moreover, the peak of implementation of the innovative technology - Normalizer devices in both segments should be expected by the beginning of the fourth year of the project (maximum of the AdoptionRate curve), and the manufacturers should be ready for this.

The main task when working with such models is to measure and set the actual market parameters presented in Table 1.

Experiments with the model make it possible to develop more informed production solutions, increasing their efficiency. The manufacturer of the innovative product can increase the market capacity by, for example, releasing devices configured for a specific customer, and constantly implementing modified devices that correspond to the development 
of SmartGrid systems, which can improve the efficiency of energy consumption management. In addition, the manufacturer of the innovative product can use the communications that arise between hotel markets and market sectors. This approach will be considered in this paper later.

\section{Conclusion}

A simulation model for predicting market behavior of the innovative patented technology based on the concept of F. Bass is studied, which concerns the division of market agents into innovators and imitators functioning under the impact of different factors. The awareness of these factors and regulated parameters allows us to identify them and effectively manage the long-term process of introducing the innovative technology to an isolated market. System dynamics as a paradigm of simulation is researched and implemented as well. The importance and complexity of the tasks of measuring and regulating market parameters used in simulation models is shown.

A methodological approach to the development of the Bass model is proposed in conditions, when the studied innovative enterprise enters new markets, introduces new business models, strategies, and communication processes.

Hypotheses about the emergence and significant development of communications between market segments are put forward and researched. The information about the dynamics of disseminating the innovative technology in one segment can influence the behavior of agents in another segment, and vice versa. The introduction of such links in the developed simulation models is natural and technically simple, but their use requires additional research.

The presented simulation models for making management decisions on the implementation of the innovative technology for managing electric energy consumption in Smart Grid systems realize simple hypotheses and use simple types of links. They can be developed to the required level of complexity.

\section{References}

1. L.Z. Feigin, S.V. Levinson, D.A. Klavsuts et al U.S. Patent \# 7816894 B2, (2007)

2. L.Z. Feigin, S.V. Levinson, I.L. Klavsuts et al, RU Patent \# 2377630 C1 (2008)

3. L.Z. Feigin RU Patent \# 120499 (2011)

4. L.Z. Feigin, S.V. Levinson, I.L. Klavsuts et al, Eurasian Patent \# 018813 (2013)

5. L.Z. Feigin, S.V. Levinson, I.L. Klavsuts et al, Ukrainian Patent \# 103498, (2013)

6. L.Z. Feigin, S.V. Levinson, I.L. Klavsuts et al, European Patent \# 2343620, (2016)

7. A.B. Klavsuts, V.G. Trubin RU Patent \# 2618115 (2017).

8. Certificate on the trademark «NORMEL» \# 43 6079, trademark owner LLC "AVEC" (2010)

9. GOST 32144-2013 Electric energy. Electromagnetic compatibility of technical equipment. Power quality limits in the public power supply systems, Standard, http://docs.cntd.ru/document/1200104301

10. EN 50160:2010 Voltage characteristics of electricity supplied by public electricity networks, Standard, http://www.cdtechnics.be/542-standard-en-50160-voltagecharacteristics-in.pdf

11. D.A. Klavsuts, I.L. Klavsuts, G.L. Rusin, Aspects of Evaluating the Efficiency of Introducing Innovative Method and Technology Demand Side Management in Smart 
Grid System, in Proceedings of the 48th International Universities' Power Engineering Conference, UPEC 2013, 2-5 September 2013, Dublin, Ireland, (2013)

12. I.L. Klavsuts, D.A. Klavsuts, G.L. Rusin, I.S. Mezhov, Perfecting business processes in electricity grids by the use of innovative technology of demand side management in the framework of the general conception of Smart Grids, in Proceedings of the 49th International Universities' Power Engineering Conference, UPEC 2014, 2-5 September 2014, Cluj-Napoca, Romania, (2014)

13. D.A. Klavsuts, I.L. Klavsuts, T.V. Avdeenko, Providing the quality of electric power by means of regulating customers' voltage, in Proceedings of the 49th International Universities' Power Engineering Conference, UPEC 2014, 2-5 September 2014, ClujNapoca, Romania, (2014)

14. I.L. Klavsuts, G.L. Rusin, D.A. Klavsuts, Managing the implementation of innovative energy saving technologies in old industrial regions of CIS and in Northern-Eastern Asian regions : experience and perspectives, Modernization of Russian economy : perspectives, paradigms, solutions, Collected papers, Novosibirsk, RF, 40-47, (2014)

15. I.L. Klavsuts, D.A. Klavsuts, A.G. Rusina, G.L. Rusin, Modes control of Smart Power Grids based on the usage of the innovative method and device of Demand Side Management, in Proceedings of the 50 International universities power engineering conference, UPEC 2015, 1-4 September 2015, Stoke-on-Trent, United Kingdom, (2015)

16. I.L. Klavsuts, G.L. Rusin, M.V. Khayrullina, Strategic models of introducing innovative technology for management of electric power consumption into world markets, in Proceedings of the the13th Intern. Scien. and Techn. Conf Actual problems of electronic instrument engineering, APEIE-2016, 11, 94-101, 3-6 Oct., Novosibirsk, $\mathrm{RF}$, (2016)

17. I.L. Klavsuts, A.G. Rusina, D.A. Klavsuts, The development of simulation model of innovative technology of AC voltage normalization for introduction into smart grid system, in Proceedings of the 51 International universities power engineering conference, UPEC 2016, 6-9 September 2016, Coimbra, Portugal, (2016)

18. A.G. Fishov, I.L. Klavsuts, N.A. Karjaubayev, D.A. Klavsuts, Decentralized smart multi-agent voltage regulation in electric grids. Ideology and modelling, in Proceedings of the 53 International universities power engineering conference, UPEC 2018, 4-7 September 2018, Glasgow, United Kingdom, (2018)

19. F. Bass, A new product growth for model consumer durables. Manag. Scien., 15, 5, 215-227 (1969)

20. J. Forrester System Dynamics: The Foundation Under Systems Thinking, Cambridge, MA 02139 (2010)

21. AnyLogic. Multi-approch simulation modeling, http://www.anylogic.com/ (2020).

22. A. Borshchev, Multi-method modelling: AnyLogic, in Discrete-Event Simulation and System Dynamics for Management Decision Making (EDS S. Brailsford, L. Churilov and B. Dangerfield), (John Wiley \& Sons Ltd, Chichester, UK, 2014)

23. S. Brailsford, L. Churilov, B. Dangerfield, (EDS) Front Matter, in Discrete-Event Simulation and System Dynamics for Management Decision Making, John Wiley \& Sons Ltd, FMatter, Chichester, UK. (2014)

24. The official site of the city of Novosibirsk, https://novo-sibirsk.ru/tocitizens/economy/business/ (reference date 21.05.2020) 